\title{
Influência do gênero em estudos de bioequivalência de medicamentos
}

\section{Gender influence in drug bioequivalence studies}

Recebido em: 06/09/2016 Aceito em: $05 / 02 / 2017$
José Wellithom Viturino da SILVA; Rafael Ferreira de LIMA; Ana Rosa Brissant de ANDRADE; Juliana KISHISHITA; Leila Bastos LEAL; Giovana Damasceno SOUSA Departamento de Ciências Farmacêuticas, Universidade Federal de Pernambuco. Av Prof Artur de Sá, s/n. Cidade Universitária, Recife, PE. CEP 50740-520. Brasil.E-mail: giovana.sousa@nudfac.com.br

\begin{abstract}
Anatomical and physiological differences between the male and female can change the pharmacokinetic process of a drug and thus interfere with its bioavailability, making a particular drug non-bioequivalent when compared in only one gender. The aim of this study was to evaluate the influence of gender on the bioequivalence study of three drug tests in coated tablet form: Methyldopa $500 \mathrm{mg}$, Diazepam $10 \mathrm{mg}$ and Scopolamine Butylbromide $10 \mathrm{mg}$. Therefore, the pharmacokinetic parameters that determine the bioequivalence, $\mathrm{ASC} 0-\mathrm{t}, \mathrm{ASC} 0-\infty$ and $\mathrm{Cmax}$, were recalculated considering the gender separately. The studies of Diazepam and Scopolamine were bioequivalent for both gender but not bioequivalent when considered only women. Methyldopa's bioequivalence study was not bioequivalent for both sex or for the genders separately. Thus, a more careful evaluation is necessary to analyze and define the bioequivalence of medicines, in order to ensure the efficiency and safety of treatments for both genders.
\end{abstract}

Keywords: Bioequivalence; Methyldopa; Diazepam; Scopolamine Butylbromide

\section{RESUMO}

As diferenças anatômicas e fisiológicas entre os gêneros masculino e feminino podem modificar o processo farmacocinético de um fármaco e assim, interferir em sua biodisponibilidade, tornando um determinado medicamento não bioequivalente quando comparado em apenas um gênero. Dito isto, o objetivo deste trabalho foi avaliar a influência do gênero na bioequivalência de três medicamentos testes na forma de comprimido revestido: Metildopa $500 \mathrm{mg}$, Diazepam $10 \mathrm{mg}$ e Butilbrometo de Escopolamina 10mg. Para tanto, os parâmetros farmacocinéticos que determinam a bioequivalência, $\mathrm{ASC}_{0-\mathrm{t}}, \mathrm{ASC}_{0-\infty}$ e Cmáx, foram recalculados considerando os gêneros em separado. Os estudos do Diazepam e Escopolamina foram bioequivalentes para ambos os sexos, mas bioinequivalentes quando considerado apenas o sexo feminino. $\mathrm{O}$ estudo de bioequivalência da Metildopa não foi bioequivalente para ambos os sexos e nem para os sexos em separado. Assim, faz se necessário ampliar as discussões sobre a forma de analisar e definir a bioequivalência de medicamentos a fim de garantir a eficiência e segurança dos tratamentos para ambos os sexos.

Palavras-chave: bioequivalência; metildopa; diazepam; butilbrometo de escopolamina

INTRODUÇÃO

O advento dos genéricos no Brasil, inserido pela Política Nacional de Medicamentos representa um marco importante do Ministério da Saúde na área de produtos farmacêuticos. A lei dos medicamentos Genéricos (Lei no 9.787/99) teve como objetivo prin- cipal estimular a concorrência, como forma de aumentar a variedade de ofertas de medicamentos no mercado, a redução de preços e consequentemente a viabilização do acesso destes medicamentos para população $(1,2)$. 
Assim, quando a patente de um medicamento de referência expira, algumas indústrias farmacêuticas solicitam autorização para produzir um medicamento equivalente ao que perdeu a patente e para que possam ser comercializados, excluindo-se as bioisenções, devem passar pelos ensaios de biodisponibilidade relativa ou bioequivalência, a fim de atestar que este medicamento é equivalente terapêutico. Podendo-se a partir de então, inferir que eles vão ser equivalentes em seus efeitos terapêuticos, segurança e qualidade $(3,4)$.

Por definição, os estudos de bioequivalência são ensaios clínicos que visam avaliar os parâmetros farmacocinéticos que interferem na biodisponibilidade de um medicamento e comparar os resultados do medicamento teste com o de referência $(5,6)$. A biodisponibilidade pode ser definida como a velocidade e a extensão com que um fármaco é absorvido e alcança seu local de ação, que pode ser avaliada por meio de medidas farmacocinéticas capazes de determinar a liberação, absorção e eliminação de um fármaco do organismo $(7,8)$

O estudo de bioequivalência tem uma grande importância para avaliação da segurança e eficácia dos medicamentos. Se ambos, medicamento teste e referência, atingem a circulação sistêmica na mesma velocidade e extensão, eles são igualmente biodisponíveis e, consequentemente, sua eficácia e segurança será equiparada, assegurando ao consumidor que o medicamento teste terá a mesma eficácia do medicamento de referência, quando em um estudo clínico com homens e mulheres saudáveis $(9,10)$.

Por outro lado, homens e mulheres, apresentam grandes diferenças metabólicas e fisiológicas entre si (11). Estudos de farmacovigilância mostram que na exposição a doses mais elevadas de fármacos, a mulher apresenta cerca de $93 \%$ de reações adversas e homens $83 \%$. As mulheres têm tendência de apresentarem maior percentagem de gordura corporal, volume plasmático e fluxo sanguíneo em seus órgãos. Além disso, o intestino na mulher é mais lento, o que influencia diretamente na absorção de fármacos administrados via oral, a função renal da mulher é aproximadamente $10 \%$ mais baixa que a do homem e os homens possuem maior quantidade de massa muscular e secreção gástrica ácida, o que interfere no tempo de esvaziamento gástrico $(12,13)$.

Por isso, o fator gênero pode ter grande influência nos estudos de bioequivalência, uma vez que as diferenças anatômicas e de fisiologia entre ambos podem modificar o processo farmacocinético de um fármaco, desde a sua absorção até sua excreção e inferir em diferenças de biodisponibilidade entre os mesmos. Assim, tais diferenças podem mudar os resultados de um estudo de bioequivalência, tornando um determinado medicamento não bioequivalente se comparado em apenas um sexo. Esse fato, pode resultar em tratamentos ineficientes ou inseguros para um ou ambos os sexos $(14,15,16)$.

Para avaliar esta influência, os medicamentos teste e referência ao serem administrados podem ser comparados frente aos parâmetros farmacocinéticos de área sob a curva (ASC), concentração plasmática máxima (Cmax), tempo de concentração máxima (Tmax), entre outros, e a partir disso avaliada a influência do fator sexo na biodisponibilidade de um medicamento (17).

Portanto, o objetivo deste trabalho foi avaliar a influência do sexo na biodisponibilidade relativa/bioequivalência de três estudos de bioequivalência realizados com comprimidos revestidos de Metildopa $500 \mathrm{mg}$, Diazepam $10 \mathrm{mg}$ e Butilbrometo de Escopolamina 10 mg, desenvolvidos pelo Centro de Bioequivalência do Núcleo de Desenvolvimento Farmacêutico e Cosmético da Universidade Federal de Pernambuco, a fim de contribuir para uma condução mais racional de estudos de bioequivalência, o que influencia diretamente a efetividade e seguranças dos tratamentos propostos.

\section{MATERIAL E MÉTODO}

Coleta de dados. Foi realizado um estudo indireto, do tipo experimental analítico com abordagem quantitativa, com o foco principal na análise do banco de dados do Centro de Bioequivalência da Universidade Federal de Pernambuco (CBIO-NUDFAC/UFPE). Os estudos de bioequivalência de Metildopa $500 \mathrm{mg}$, Diazepam 10 $\mathrm{mg}$ e de Butilbrometo de Escopolamina $10 \mathrm{mg}$, foram realizados pelo NUDFAC entre 2011 e 2016, utilizando voluntários hígidos, não fumantes, de ambos os sexos, com idade entre 18 e 50 anos e que moravam na região metropolitana do Recife.

Parâmetros analisados. Os parâmetros farmacocinéticos $\mathrm{ASC}_{0-\mathrm{t}}$ (área sob a curva, concentração versus tempo, de zero até o tempo da última quantificação), $\mathrm{ASC}_{0-\infty}$ (área sob a curva de zero a infinito, extrapolação dos dados), e o Cmax (concentração máxima avaliada), além do poder descritivo de teste, dos estudos de bioequivalência média de comprimidos revestidos de Metildopa $500 \mathrm{mg}$, Diazepam $10 \mathrm{mg}$ e de Butilbrometo de Escopolamina $10 \mathrm{mg}$, foram recalculados, considerando os sexos em separado. Os estudos de biodisponibilidade relativa/comparativa foram abertos, cruzados e na 
condição de jejum. Aldomet ${ }^{\circledR} 500 \mathrm{mg}$, Valium ${ }^{\circledR} 10 \mathrm{mg}$ e Buscopan ${ }^{\circledR} 10 \mathrm{mg}$, foram os medicamentos referência utilizados nas comparações, todos os medicamentos testes eram similares industrializados.

Tratamento estatístico. Os dados dos parâmetros de Cmax e ASC foram transformados logaritmicamente, uma vez que, em geral, os dados transformados aproximam-se de uma distribuição normal em relação aos dados originais. Uma análise de variância (ANOVA) foi empregada aos dados transformados dos parâmetros farmacocinéticos Cmax e ASC, por meio de modelos estatísticos apropriados; com isso foi criada uma tabela contendo o grau de liberdade, soma dos quadrados, quadrado médio, estatística $F$, valor de p, para avaliação dos efeitos de sequência, voluntário dentro de sequência, período e tratamento e os coeficientes de variação intra e interindividuais. Para a avaliação de efeitos de sequência, foi utilizado um nível de significância correspondente a $10 \%$ (valor de $\mathrm{p}<0.10$ ), e para os demais efeitos o nível de significância utilizado foi $5 \%$ (valor de $\mathrm{p}<0.05$ ) (18). O processamento e a análise dos dados foram realizados utilizando o WinNonLin Professional Network Edition version 5.2 e Microsoft Excel Version 7.0.

Valores de aceitação. Dois medicamentos foram considerados bioequivalentes quando os valores extremos do intervalo de $90 \%$ de confiança, para a razão das médias geométricas dos parâmetros Cmax e ASC, estiveram dentro dos limites de aceitação recomendado pela agência reguladora, isto é, maiores que 0,8 e menores que 1,25 (19)

Aspectos éticos. Todos os estudos utilizados nesta pesquisa foram conduzidos de acordo com a Declaração de Helsinki (1965) e suas revisões, além da Resolução 466/12 do Conselho Nacional de Saúde e aprovados pelo Comitê de Ética em Pesquisa da UFPE, pareceres de número 98.666, 340.557 e 215.923. Os dados utilizados para análise são confidenciais e o anonimato dos sujeitos envolvidos foi assegurado.

\section{RESULTADOS E DISCUSSÃO}

Os estudos de bioequivalência analisados foram realizados de acordo com a RE $\mathrm{n}^{\circ} 1170$ de 2006, da Agência Nacional de Vigilância Sanitária (19), que traz as exigências para o desenvolvimento de trabalhos com biodisponibilidade relativa/bioequivalência de medicamentos. O número de voluntários do estudo está diretamente ligado ao nível de significância estabelecido para o estudo, da variabilidade do fármaco, bem como do poder do teste.

As Tabelas 1 a 3 trazem os resultados dos parâmetros farmacocinéticos avaliados para definir a bioequivalência dos medicamentos testes, considerando o estudo para ambos os sexos e para os sexos em separado. A partir disso pode ser avaliada a influência dos sexos na biodisponibilidade relativa desses medicamentos..

Tabela 1: Parâmetros farmacocinéticos do estudo de bioequivalência em comprimidos revestidos de Butilbrometo de Escopolamina $10 \mathrm{mg}$ para ambos os sexos e para os sexos em separado (IC ${ }_{90 \%}$ )

\begin{tabular}{|c|c|c|c|c|c|}
\hline Parâmetro & $\mathrm{N}$ & Razão \% & Limite inferior \% & Limite superior \% & Poder \% \\
\hline$C_{\max (A S)}$ & 58 & 106.39 & 94.96 & 119.19 & 94.36 \\
\hline $\mathrm{ASC}_{0-\mathrm{t}(\mathrm{AS})}$ & 58 & 109.76 & 97.90 & 123.06 & 94.14 \\
\hline $\mathrm{ASC}_{0 \text {-inf (AS) }}$ & 58 & 110.09 & 98.47 & 123.08 & 95.02 \\
\hline$C_{\max (S M)}$ & 30 & 101.79 & 91.61 & 117.06 & 85.28 \\
\hline $\mathrm{ASC}_{0-t(S M)}$ & 30 & 105.48 & 92.82 & 121.32 & 88.69 \\
\hline$A S C_{0 \text {-inf(SM) }}$ & 30 & 104.98 & 92.38 & 118.63 & 89.12 \\
\hline $\mathrm{C}_{\max (\mathrm{SF})}$ & 28 & 111.18 & 94.51 & 112.30 & 79.58 \\
\hline $\mathrm{ASC}_{0-\mathrm{t}(\mathrm{SF})}$ & 28 & 117.06 & 100.67 & 128.76 & 80.27 \\
\hline$A S C_{0 \text {-inf(SF) }}$ & 28 & 118.10 & 100.38 & 134.63 & 80.69 \\
\hline
\end{tabular}

N: número de voluntários; AS: ambos os sexos; SM: sexo masculino; SF: sexo feminino. 


\section{lnoparma}

Tabela 2: Parâmetros farmacocinéticos do estudo de bioequivalência de comprimidos revestidos de Diazepam $10 \mathrm{mg}$ para ambos os sexos e para os sexos em separado(IC ${ }_{90 \%}$ ).

\begin{tabular}{|c|c|c|c|c|c|}
\hline Parâmetro & N & Razão\% & Limite inferior \% & Limite Superior \% & Poder \% \\
\hline$C_{\max (A S)}$ & 34 & 95.32 & 85.39 & 106.41 & 95.44 \\
\hline ASC $_{0-\text { t(AS) }}$ & 34 & 100.77 & 96.21 & 105.55 & 99.99 \\
\hline ASC $_{0 \text {-inf (AS) }}$ & 34 & 100.07 & 91.19 & 109.81 & 98.81 \\
\hline$C_{\operatorname{max(SM)~}}$ & 17 & 93.063 & 80.38 & 109.10 & 75.53 \\
\hline ASC $_{\text {0-t(SM) }}$ & 17 & 103.68 & 97.59 & 110.15 & 99.99 \\
\hline ASC $_{\text {0-inf(SM) }}$ & 17 & 104,23 & 89.15 & 121.87 & 76.80 \\
\hline$C_{\operatorname{max(SF)~}}$ & 17 & 97.44 & 83.36 & 113.88 & 79.93 \\
\hline ASC $_{\text {0-t(SF) }}$ & 17 & 102.86 & 91.28 & 115.91 & 92.59 \\
\hline ASC $_{0-\text {-inf(SF) }}$ & 17 & 141.38 & 67.80 & 294.81 & 13.91 \\
\hline
\end{tabular}

N: número de voluntários; AS: ambos os sexos; SM: sexo masculino; SF: sexo feminino.

Tabela 3: Parâmetros farmacocinéticos do estudo de bioequivalência de comprimidos revestidos de metildopa $500 \mathrm{mg}$ para ambos os sexos e para os sexos em separado (IC ${ }_{90 \%}$ ).

\begin{tabular}{|c|c|c|c|c|c|}
\hline Parâmetro & N & Razão\% & Limite inferior \% & Limite Superior \% & Poder \% \\
\hline$C_{\max (A S)}$ & 34 & 118.40 & 104.27 & 134.44 & 89.55 \\
\hline ASC $_{\text {0-t(AS) }}$ & 34 & 116.22 & 104.17 & 129.67 & 95.58 \\
\hline ASC $_{0 \text {-inf (AS) }}$ & 34 & 116.13 & 104.06 & 129.59 & 95.53 \\
\hline$C_{\operatorname{max(SM)}}$ & 17 & 111.54 & 97.03 & 126.04 & 75.24 \\
\hline ASC $_{0-\text { t(SM) }}$ & 17 & 113.71 & 98.92 & 130.76 & 78.69 \\
\hline ASC $_{0 \text {-inf(SM) }}$ & 17 & 113.58 & 99.95 & 128.21 & 79.12 \\
\hline$C_{\max (S)}$ & 17 & 124.13 & 104.26 & 143.99 & 69.88 \\
\hline ASC $_{0-\text { t(SF) }}$ & 17 & 117.18 & 98.92 & 134.76 & 72.58 \\
\hline ASC $_{0-\text {-inf(SF) }}$ & 17 & 117.2 & 99.45 & 135.95 & 72.02 \\
\hline
\end{tabular}

N: número de voluntários; AS: ambos os sexos; SM: sexo masculino; SF: sexo feminino.

Os parâmetros utilizados foram o Cmax, ASC0-t e ASC0-inf, com intuito de determinar o intervalo de confiança de 90\% (I.C. 90\%) para as relações entre Cmax e ASC0-t dos produtos testes e referência, na escala logarítmica, sendo o medicamento considerado bioequivalente quando os parâmetros farmacocinéticos supracitados apresentarem valores entre 80 e $125 \%$ em relação ao medicamento de referência (19).

O número de voluntários incluídos nos estudos analisados apresentou o número mínimo de 12 , conforme exigido pela Anvisa. No entanto, é importante salientar que a redução do número de sujeitos utilizados para a análise de bioequivalência, considerando os sexos em separado, tende a reduzir o poder de teste e a probabilidade de bioequivalência entre os medicamentos teste e referência. De um modo geral, o poder de teste dos diferentes estudos apresentaram valores próximos ou acima de $80 \%$, exceto para o parâmetro de $\mathrm{ASC}_{0 \text {-inf(SF) }}$ trazido na avaliação de bioequivalência do Diazepam $10 \mathrm{mg}$ e $\mathrm{C}_{\max (\mathrm{SF})}$ trazido na avaliação de bioequivalência de Metildopa $500 \mathrm{mg}$ (Tabela 1). 
A Escopolamina é um antagonista do receptor muscarínico não específico, utilizado principalmente para alívio da dor e desconforto abdominal (20). Nos estudos de bioequivalência de duas formulações de butilbrometo de escopolamina, na forma de comprimido revestido de $10 \mathrm{mg}$, considerando 58 voluntários de ambos os sexos e, considerando apenas o sexo masculino (30 voluntários), o medicamento teste foi considerado bioequivalente ao medicamento de referência $\left(\right.$ Buscopan $^{\circledR}$ ), pois todos os parâmetros farmacocinéticos avaliados estavam dentro dos limites especificados pela RE 1170 de 2006 da Anvisa (19), conforme pode ser verificado na Tabela 1.

Quando considerado apenas o sexo feminino, utilizando 28 voluntárias, o medicamento teste foi considerado não-bioequivalente, porque o limite superior do índice de confiança de $90 \%$ para o $\mathrm{ASC}_{0-\mathrm{t}}$ e $\mathrm{ASC}_{0 \text {-inf }}$ estava fora da faixa de 80 A $125 \%$ (Tabela 3 ).

Além disso, o poder de teste no estudo de bioequivalência da escopolamina, quando foram avaliados ambos os sexos e os sexos separados, apresentou valores quase sempre superiores a $80 \%$, o que reflete na probabilidade do estudo em identificar uma diferença entre os tratamentos e por consequência na confiabilidade dos resultados obtidos (21).

Da mesma forma, o estudo de bioequivalência realizado para o medicamento teste de diazepam, um dos ansiolíticos benzodiazepínicos mais utilizados no país (22), demonstrou que o mesmo, embora bioequivalente em relação ao medicamento referência $\left(\right.$ Valium $\left.^{\circledR}\right)$ para ambos os sexos - no estudo com 34 participantes - e para o sexo masculino - 17 voluntários - foi bioinequivalente para o sexo feminino (Tabela 2).

No estudo de bioequivalência do medicamento teste de metildopa $500 \mathrm{mg}$, medicamento amplamente utilizado no controle da hipertensão arterial de severidade moderada a grave, a amostra não foi considerada bioequivalente para ambos os sexos e, tampouco, para os sexos em separado, pois o limite superior do índice de confiança de $90 \%$ para o Cmax, $\mathrm{ASC}_{0-\mathrm{t}}$ e $\mathrm{ASC}_{0 \text {-inf }}$ estava fora da faixa de 80 a $125 \%$ (20), para todos os casos avaliados (Tabela 3).

Os resultados destes estudos mostraram que as diferenças entre sexos podem influenciar o resultado da bioequivalência de um medicamento, interferindo assim, na eficiência e segurança dos tratamentos propostos, principalmente, para o sexo feminino. Assim, vários autores têm expressado a necessidade para a inclusão científica e a análise do impacto do sexo na pesquisa clínica $(23,24)$, sendo, portanto, um tema de interesse atual e incontornável numa sociedade em que se pretende que as terapias individualizadas assumam uma importância crescente.

Alguns fatores podem contribuir para o entendimento de como o sexo influencia na biodisponibilidade de um medicamento. Estudos anteriores demonstraram que o risco de reações adversas a fármacos, no sexo feminino, pode atingir valores superiores quando comparados ao sexo masculino (6). Homens e mulheres apresentam diferenças antropométricas significativas que podem interferir na absorção e distribuição de um fármaco (25). Estudos de farmacovigilância mostraram um maior percentual de eventos adversos, associados à dose administrada, em mulheres, corroborando a importância do fator peso corporal, o trânsito gastrointestinal, de uma forma geral, é mais lento na mulher que no homem, o que poderá ter importância na absorção de fármacos administrados por via oral $(26,27,28)$.

No que diz respeito à função renal, homens e mulheres também apresentam algumas diferenças. As mulheres apresentam uma taxa de filtração glomerular menor do que os homens, e os homens tendem a ter níveis de creatinina plasmática mais elevados porque possuem maior quantidade de massa do músculo esquelético. $\mathrm{O}$ fígado que é um dos mais importantes na modulação dos efeitos dos fármacos também apresenta significativa diferença entre os sexos (11).

Outro parâmetro bastante observado, e que tem uma grande influência, é o fator hormonal. Hormônios sexuais podem influenciar secreção ácida gástrica, a motilidade e tempo de trânsito gastrointestinal, percentagem de tecido adiposo, de massa muscular e de água corporal, entre outros $(29,30)$. Os hormônios sexuais podem interferir na farmacocinética e, consequentemente, na farmacodinâmica contribuindo para o aparecimento de diferentes respostas para um mesmo tratamento, entre os sexos. A composição hormonal apresenta uma relação bidirecional com os fármacos, uma vez que não só as hormonas podem influenciar o fármaco, como o próprio fármaco pode influenciar os níveis ou ações hormonais (31).

\section{CONCLUSÃO}

De acordo com os resultados obtidos, pode ser concluído que os medicamentos Diazepam e Butilbrometo de Escopolamina que, inicialmente, no estudo de bioequivalência para ambos os sexos se apresentaram bioequivalentes, as diferenças fisiológicas entre os se- 
xos promoveram interferência nos resultados da biodisponibilidade relativa, não sendo, nas condições avaliadas, bioequivalentes, quando considerado apenas o sexo feminino. Ainda, para o estudo de bioequivalência de metildopa $500 \mathrm{mg}$, que não foi bioequivalente para o estudo misto e, tampouco, para os sexos em separado, pode ser inferido que a bioinequivalência não está diretamente relacionada com as diferenças entre os sexos e, provavelmente, relaciona-se com diferenças biofarmacotécnicas entre as formulações dos medicamentos teste e referência. Por fim, é necessário estender as discussões sobre o impacto das diferenças fisiológicas entre sexos nas pesquisas clínicas, em especial nos estudos de bioequivalência.

\section{AGRADECIMENTOS}

Ao Núcleo de Desenvolvimento Farmacêutico e Cosmético (NUDFAC/ UFPE), pelo apoio no desenvolvimento deste trabalho.

\section{REFERÊNCIAS}

1. Bedor DCG. O novo contexto da intercambialidade de medicamentos no Brasil: Medicamentos Similares e suas bases técnico-cientificas. Boletim Informativo Geum. 5 (2):7-13, 2014; DOI: 10.13140/RG.2.1.2336.1444.

2. Rumel D, Nishioka AS, Santos AAM. Intercambialidade de medicamentos: abordagem clínica e o ponto de vista do consumidor.Rev Saude Publica. 2006; 40 (5): 921927. DOI: 10.1590/S0034-89102006000600024.

3. Araújo LU, Albuquerque KTD, Kato KC, Silveira GS, Maciel NR, Spósito PÁ, Storpirtis S. Medicamentos genéricos no Brasil: panorama histórico e legislação. Rev Panam Salud Publica. 2010; 28 (6): 480-92.

4. Lopes RA, Neves FA R. Metanálise de estudos de bioequivalência: a intercambiabilidade de genéricos e similares que contêm Hidroclorotiazida é possível, mas não àqueles com Maleato de Enalapril. J Bras Nefrol. 2010; 32 (2): 173-181. DOI: 10.1590/S0101-28002010000200006.

5. Kuribayashi R, Takishita T, Mikami K. Regulatory Considerations of Bioequivalence Studies for Oral Solid Dosage Forms in Japan. J Pharm Sci. 2016; 105 (8): 22702277. DOI: $10.1016 /$ j.xphs.2016.05.026.

6. Holdcroft A. Gender bias in research: how does it affect evidence based medicine? J R Soc Med. 2007; 100 (1): 2-3. DOI: $10.1258 / \mathrm{jrsm} .100 .1 .2$.

7. Manjunath K, Venkateswarlu V. Pharmacokinetics, tissue distribution and bioavailability of clozapine solid lipid nanoparticles after intravenous and intra duodenal administration. J Control Release. 2005; 107 (2): 215-228. DOI: 10.1016/j.jconrel.2005.06.006.

8. Diletti E, Hauschke D, Steinijans VW. Sample Size Determination for Bioequivalence by Means of Confidence Intervals. Int J Clin Pharmacol Ther. 1991; 29 (1): 1-8. DOI:10.1007/BF01061471.

9. Herrera-Calderón O, Grande-Ortiz M. Equivalencia terapéutica de tabletas de diazepam dispensadas em laciudad de Ica, Perú. Rev Méd Herediana. 2012; 23 (3): 154- 159. DOI:10.20453/rmh.v23i3.1023.

10. Davit BM, Nwakama PE, Buehler GJ, Conner DP, Haidar SH, Pate LDT, Woodcock J. Comparing generic and

innovator drugs: a review of 12 years of bioequivalence data from the United States Food and Drug Administration. Ann Pharmacother. 2009; 43 (10): 1583-1597. DOI: 10.1345/aph.1M141.

11. Schwartz, J. B. The influence of sex on pharmacokinetics. Clinical pharmacokinetics. 2003; 42 (2): 107-121. DOI: $10.2165 / 00003088-200342020-00001$.

12. Soldin OP, Mattison DR. Sex differences in pharmacokinetics and pharmacodynamics. Clinical pharmacokinetics. 2009; 48 (3): 143-157. DOI:10.2165/00003088200948030-00001.

13. Hauschke D, Steinijans VW, Pigeot I. Bioequivalence Studies in Drug Development - Methods and Applications. Chichester: John Wiley \& Sons Ltd. 2007.

14. Ibarra M, Magallanes L, Lorier M, Vázquez M, Fagiolino P. Sex-by-formulation interaction assessed through a bioequivalence study of efavirenz tablets. Eur J Pharm Sci. 2016; 85: 106-111. DOI: 10.1016/j.ejps.2016.02.001.

15. Franconi F, Brunelleschi S, Steardo L, Cuomo V. Gender differences in drug responses. Pharmacol Res. 2007; 55 (2): 81-95. DOI: 10.1016/S0074-7742(08)00001-9.

16. Schwartz J B. The current state of knowledge on age, sex, and their interactions on clinical pharmacology. Clin Pharm Ther. 2007; 82 (1): 87-96. DOI: 10.1038/sj. clpt.6100226.

17. Moreno Exebio L. Aspectos éticos de los estudios de biodisponibilidad y bioequivalencia de produtos farmacêuticos contenidos em las legislaciones de América Latina. Acta Bioeth. 2004; 10(2): 247-259. DOI: 10.4067/ S1726-569X2004000200012.

18. Chow SC, Liu JP. Design and Analysis of Bioavailability and Bioequivalence Studies. 2nd. ed. New York: Marcel Dekker. 2000.

19. BRASIL. Ministério da saúde. A Agência Nacional de Vigilância Sanitária. Resolução Específica (RE) n ${ }^{\circ}$ 1170, de 19 de abril de 2006. Determinar a publicação do Guia para provas de biodisponibilidade relativa/bioequivalência de medicamentos. Diário da União Poder Executivo, Brasília, 24 abril. 2006. 13 p. 
20. Seifhosseini S, Jahanshahi M, Moghimi A, Aazami N The Effect of Scopolamine on Avoidance Memory and Hippocampal Neurons in Male Wistar Rats. J Clin Neurosci. 2011; 3 (1): 9-15.

21. Coutinho ESF, Da Cunha GM. Conceitos básicos de epidemiologia e estatística para a leitura de ensaios clínicos. Rev Bras Psiquiatr. 2005; 27 (2): 146-151. DOI 10.1590/ S1516-44462005000200015.

22. Goodman, Gilman: As Bases Farmacológicas da Terapêutica. 12th. ed. Rio de Janeiro: Mc Graw-Hill. 2012.

23. Franconi F, Sanna M, Straface E \& Chessa R. IN: Phamacokinetics and pharmacodynamics: the role sex and gender. London: Springer London, 2012. p. 183-194.

24. Geller SE, Adams MG, Carnes M. Adherence to federal guidelines for reporting of sex and race/ethnicity in clinical trials. J Women Health (Larchmt). 2006; 15 (10): 1123-1131. DOI: 10.1089/jwh.2006.15.1123

25. Anderson GD. Gender differences in pharmacological response. Int Rev Neurobiol. 2008; 83:1-10. DOI: 10.1016/S0074-7742(08)00001-9.
26. González N, Fagiolino V, Vàzquez M, Eiraldi R. Bioequivalence apromedio teniendo en cuentael género de lossujetos. Lat Am J Pharm. 2009; 28 (10): 1123-1131

27. Rubinow DR \& Moore M. Sex-dependent modulation of treatment response. Dialogues Clin Neurosci. 2004; 6 (1): 39-51.

28. Rademaker M. Do Women Have More Adverse Drug Reactions? Am J Clin Dermatol. 2001; 2 (6): 349-351. DOI: 10.2165/00128071-200102060-00001.

29. Regitz-Zagrosek V. Sex and Gender Differences in Pharmacology. New York: Springer. 2012.

30. García-Arieta A, Gordon J. Bioequivalence requirements in the European Union: critical discussion. AAPS J. 2012; 14 (4): 738-748. DOI: 10.1208/s12248-012-9382.

31. Frank A, Brown LM, Clegg DJ. The role of hypothalamic strogen receptors in metabolic regulation. Front Neuroendocrinol. 2014; 35 (4): 550-557. DOI: 10.1016/j. yfrne.2014.05.002. 